\title{
STEM-EELS Study of Plasmonic Modes in Ag nanotriangles: Size and Dielectric Dependence.
}

\author{
R. Sachan ${ }^{1}$, M. A. Roldan ${ }^{1,4}$, D. Jin' ${ }^{2}$, W. J. Weber ${ }^{3,1}$ and N. X. Fang ${ }^{2}$ \\ 1. Materials Science and Technology Division, Oak Ridge National Laboratory, Oak Ridge TN \\ 37831, USA \\ 2. Department of Mechanical Engineering, Massachusetts Institute of Technology, Cambridge MA \\ 02139, USA. \\ 3. Department of Materials Science and Engineering, University of Tennessee, Knoxville TN \\ 37996, USA. \\ 4. Imaging and Characterization Core Lab, KAUST, Thuwal 23955, KSA
}

Localized surface plasmon resonance (LSPR) have been probe to be very useful in many applications like detection of single viruses and single biological nano-objects[1], or improve photovoltaic devices[2] among others. The LSPR excitations in a nanomaterial can be altered by many factors from particle geometry to ambient environment dielectric, and further can be tuned for specific applications.

In this work, we study the quality factor of each surface plasmonic mode excited on $\mathrm{Ag}$ nanotriangles which is the measure of materials acceptibilty for plasmonics based devices. Present work is focus on triangular shaped Ag nanoparticles, which have a high quality factor and a simple geometry to ease the understanding the complicated physics of plasmonic excitations [3]. In addition, the LSPR modes in Ag lies in the visible and infrared light range which makes in compelling candidate for the development of highly sensitive photonic and phononic biosensors [4].

It is shown in the study that the triangle shape nanoparticles show three surface plasmon modes, at (i) corners, (ii) edge center and (iii) entire edge[5]. In the present work, we will study how LSPs get affected by changing the nanoparticle size and the surrounding dielectric media. In order to be able to study this effect Ag nanotriangles were synthesized and then coated with $20 \mathrm{~nm}$ thin films of $\mathrm{HfO}_{2}(\varepsilon=25)$ and $\mathrm{TiO}_{2}(\varepsilon=86)$. The whole study has been done using electron energy-loss spectroscopy (EELS) technique in a monochromator equipped scanning transmission electron microscope (STEM) with an energy resolution $(0.15 \mathrm{eV})$. With this tool, we were able to excite the three plasmonic modes and study the dielectric sensitivity in silver nanotriangles with high spatial and energy resolution. 
References:

[1] S. Victoria, Application of Surface Plasmon Resonance (SPR) for the Detection of Single Viruses and Single Biological Nano-objects, J. Bacteriol. Parasitol. 03 (2012).

[2] H.A. Atwater, A. Polman, Plasmonics for improved photovoltaic devices., Nat. Mater. 9 (2010) 205-213.

[3] M.G. Blaber, M.D. Arnold and M.J. Ford, A review of the optical properties of alloys and intermetallics for plasmonics, J. Phys. Condens. Matter. 22 (2010) 143201.

[4] H. Wei, S.M. Hossein Abtahi and P.J. Vikesland, Plasmonic colorimetric and SERS sensors for environmental analysis, Environ. Sci. Nano. 2 (2015) 120-135.

[5] J. Nelayah et al., Mapping surface plasmons on a single metallic nanoparticle, Nat. Phys. 3 (2007) 348-353.

[6] This work was supported by the U.S. Department of Energy, Office of Science, and Basic Energy Sciences, Materials Sciences and Engineering Division.
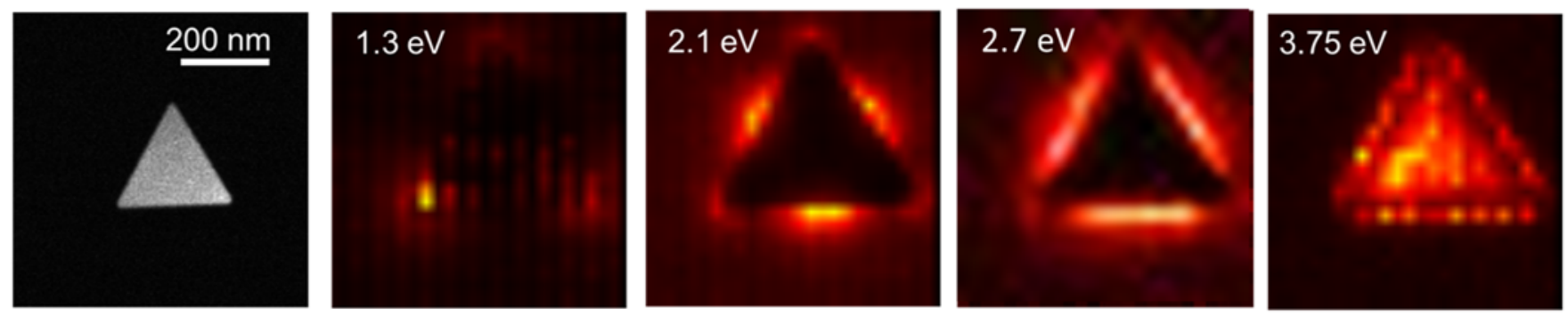

Figure 1. Ag nanotriangle and the exhibited plasmon modes at different energies. Plasmon modes are preferentially excited at selected region of the nanotriangle. 